\title{
Escala de Positividade (EP): Novas Evidências de Validade no Contexto Brasileiro
}

\author{
Juliane Callegaro Borsa - Pontifícia Universidade Católica do Rio de Janeiro, Rio de Janeiro, Brasil \\ Bruno Figueiredo Damásio - Universidade Federal do Rio Grande do Sul, Porto Alegre, Brasil \\ Silvia Helena Koller - Universidade Federal do Rio Grande do Sul, Porto Alegre, Brasil
}

\begin{abstract}
Resumo
O presente estudo investiga novas evidências de validade para a Escala de Positividade (EP) para o contexto brasileiro. Participaram deste estudo 876 indivíduos (66,0\% mulheres), com idades variando entre 18 e 83 anos. Uma Análise Fatorial Confirmatória Multigrupo foi conduzida para avaliar a invariância da EP para sexo. Os resultados indicaram invariância configural, métrica e escalar, demostrando que a EP pode ser utilizada indistintamente para homens e mulheres. Duas análises de regressão linear múltipla foram realizadas para avaliar a relação da EP com variáveis constituintes do construto positividade (modelo 1), e com variáveis convergentes (modelo 2). Os resultados do modelo 1 demonstraram que, de fato, a positividade pode ser considerada um compósito das variáveis autoestima, esperança, otimismo e satisfação com a vida. Os resultados do segundo modelo atestaram a validade convergente da escala com outras medidas correlatas. Este estudo amplia as evidências de validade da EP no contexto brasileiro.
\end{abstract}

Palavras-chave: positividade, psicometria, evidências de validade, escala

Positivity Scale (POS): New Evidence of Validity in the Brazilian Context

\begin{abstract}
This study investigates new evidence of validity for the Positivity Scale (POS) to the Brazilian context. 876 individuals $(66 \%$ women), with age varying from 18 to 83 years old participated in the study. A multigroup confirmatory factor analysis was conducted to evaluate gender measurement invariance. Results showed configural, metric and scalar invariance, suggesting POS to be invariant for both men and women. Two multiple linear regression analysis were conducted to evaluate the relation among the POS with constituent variables of the construct positivity (model 1) and with convergent constructs (model 2). Results from model 1 suggested that, in fact, positivity can be considered a composite of self-esteem, hope, optimism and life satisfaction. Results from model 2 provided further convergent validity evidence for the scale. This study extends the validity evidence for the POS in the Brazilian context.

Keywords: positivity, psychometrics, evidence of validity, scale
\end{abstract}

Escala de Positividad (EP): Nuevas Evidencias de Validez en el Contexto Brasileño

\begin{abstract}
Resumen
El presente estudio investiga nuevas evidencias de validez de la Escala de Positividad (EP) en el contexto brasileño. Los participantes fueron 876 individuos (66\% mujeres) con edades entre 18 y 83 años. Un Análisis Factorial Confirmatorio Multigrupo fue realizado para evaluar la invariancia de la EP con respecto al sexo. Los resultados mostraran invariancia configural, métrica y escalar, demostrando que la EP puede ser utilizada indistintamente por hombres y mujeres. Dos análisis de regresión lineal múltiple fueron realizados para evaluar la relación de la EP con variables constituyentes del constructo positividad (modelo 1) y con variables convergentes (modelo 2). Los resultados del modelo 1 mostraron que la positividad puede ser considerada un compuesto de las variables autoestima, esperanza, optimismo y satisfacción con la vida. Los resultados del modelo 2 atestiguaron la validez convergente de la escala con otras medidas relacionadas. Este estudio amplía las evidencias de validez de la EP en el contexto brasileño.

Palabras clave: positividad, psicometría, evidencias de validez, escala
\end{abstract}

Nas últimas décadas vem-se observando um crescente interesse acerca das características psicológicas positivas que predispõem os indivíduos a vivenciarem plenamente suas potencialidades, resultando em uma vida exitosa para si e para aqueles com quem se relacionam (Fredrickson, 2009; Gable \& Haidt, 2005; Seligman \& Csikszentmihalyi, 2000). Dentre os principais construtos investigados estão o otimismo (Carver, Scheier, \& Segerstrom, 2010), a esperança (Snyder, Rand, \& Sigmon, 2002), a felicidade (Lyubomirski \& Lepper, 1999), a resiliência

Disponivel em www.scielo.br
(Werner, 2013), bem-estar subjetivo (Diener, 2013) e, mais recentemente a positividade (Fredrickson, 2009; Jayawickreme \& Pawelski, 2012). Esta última, também denominada "pensamento positivo" (Caprara \& Steca, 2005; 2006; Scheier \& Carver, 1993) ou "orientação positiva" (Caprara et al., 2012a, 2012b) refere-se à disposição geral do indivíduo para avaliar positivamente os diversos aspectos da vida (Diener, Scollon, Oishi, Dzokoto, \& Suh, 2000). Estudos têm demonstrado que a positividade apresenta associação negativa com afetos negativos e depressão, e associação positiva 
com afetos positivos, qualidade de amizades, extroversão, amabilidade, resiliência, bem-estar psicológico, crenças de autoeficácia e estabilidade emocional (Alessandri, Caprara, \& Tisak, 2012; Alessandri et al., 2012; Caprara, Alessandri, \& Barbaranelli, 2010).

A positividade influencia o comportamento e as reações frente aos eventos estressores e a crença que os indivíduos têm acerca do futuro (Fredrickson, 2009; Scheier e Carver,1993). Estudos longitudinais têm demonstrado que a positividade é uma característica relativamente estável, variando pouco entre a adolescência e a idade adulta (Alessandri, Caprara, \& Tisak, 2011). Em termos de definição do construto, a positividade refere-se a um traço latente que explica as variações e a estabilidade quanto aos níveis de autoestima, satisfação com a vida, otimismo e esperança, apesar das mudanças ocorridas no ambiente (Caprara et al., 2012a; 2012b; Caprara \& Steca, 2005; 2006; Caprara, Steca, Alessandri, Abela, \& McWhinnie, 2010). Estudos realizados com gêmeos monozigóticos têm demonstrado, ainda, que fatores genéticos seriam capazes de explicar a alta covariância entre estes construtos (variando entre 0,80 e 0,87 ), sugerindo haver um traço latente único (positividade), com forte influência genética que representaria o conjunto dessas variáveis (Caprara et al., 2009; Caprara, 2012, julho).

Com o objetivo de avaliar os níveis de positividade em indivíduos adultos, Caprara et al., (2012 ; 2012b) desenvolveram a Escala de Positividade (EP), em inglês Positivity Scale ou P-Scale. Trata-se de um questionário composto por oito itens que avaliam a visão positiva que as pessoas têm sobre si mesmas, sobre a vida e sobre o futuro. A EP tem sido utilizada em diferentes contextos culturais, apresentando invariância estrutural em países como Itália, Canadá, Alemanha e Japão (Caprara et al., 2012a; 2012b).

No Brasil, a EP foi traduzida, adaptada e validada por Borsa, Damásio, Souza, Koller e Caprara (2015) em uma amostra de 730 indivíduos (65\% mulheres), com idades entre 17 e 70 anos de idade $(M=31,0$ anos, $D P$ $=11,43)$, residentes em 21 estados brasileiros. Análises fatoriais exploratórias e confirmatórias indicaram a unidimensionalidade do instrumento, corroborando a estrutura da versão original (Caprara et al., 2012a). Este estudo indicou correlação positiva entre os escores da EP e os escores obtidos em instrumentos de avaliação de saúde mental, sentido de vida, felicidade subjetiva e satisfação com a vida. Os escores de positividade na amostra brasileira apresentaram baixa correlação positiva com as variáveis Idade, Escolaridade e Renda mensal. Associações com baixo tamanho de efeito foram encontradas para as variáveis Empregabilidade e Estado civil. Os resultados sugeriram que a positividade parece estar mais relacionada às características individuais do que aos aspectos sociodemográficos, corroborando as hipóteses de Caprara et al. (Caprara et al., 2009; 2010; Caprara, 2012, julho).

O estudo desenvolvido por Borsa et al. (2015) permitiu encontrar evidências iniciais de validade baseadas na estrutura interna da EP e baseadas nas relações com outras variáveis. No entanto, conforme sugere o Standards for Educational And Psychological Testing (AERA; APA; NCME, 1999), a validade de um instrumento é entendida como "o grau em que todas as evidências acumuladas corroboram a interpretação pretendida dos escores de um teste para a finalidade a que se propõe" (AERA; APA; NCME, 1999, p. 11). Nesse sentido, entende-se que novos estudos são necessários, na medida em que permitem confirmar e/ou acrescentar evidências de validade para a EP. Conforme aponta Urbina (2004), é necessária uma ampla fonte de evidências de validade para considerar um instrumento válido em um determinado contexto.

O presente estudo teve por objetivo investigar novas evidências de validade para a EP. Especificamente, buscou-se avaliar a invariância da medida para homens e mulheres, bem como apresentar novas evidências de validade de construto para o instrumento. Espera-se que os resultados corroborem as evidências de que a positividade, conforme medido pela EP, seja um compósito das variáveis Autoestima, Esperança, Otimismo e Satisfação com a vida. Do mesmo modo, espera-se que outras variáveis positivas (i.e., Esperança, Sentido de vida, Vitalidade, Autoeficácia, Estresse e Felicidade) apresentem associação com a positividade, conforme apresentado em estudos anteriores.

\section{Método}

\section{Participantes}

Participou deste estudo uma amostra de 876 indivíduos adultos $(66,0 \%$ mulheres; $n=578)$, com idade variando entre 18 e 83 anos $(M=32,09 ; D P=10,98)$. Do total de participantes, $57 \%$ residiam na região Sul; $12,6 \%$, na região Sudeste; $10 \%$, na região Centro-Oeste; $17,2 \%$, na região Nordeste e 3,2\%, na Região Norte. No momento da pesquisa, $61,4 \%$ declararam estar solteiros; $32,4 \%$, casados; $5,5 \%$, separados ou divorciados e $0,7 \%$, viúvos (Tabela 1 ). 
Tabela 1

Características Sociodemográficas da Amostra

\begin{tabular}{|c|c|c|c|}
\hline \multirow{2}{*}{ Varável } & Total & Homens & Mulheres \\
\hline & $N=876$ & $n=298(34 \%)$ & $n=578(66 \%)$ \\
\hline Idade M (DP) & $39,30(16,89)$ & $32,55(11,36)$ & $31,19(10,18)$ \\
\hline Amplitude de idade & $18-83$ & $18-83$ & $18-71$ \\
\hline \multicolumn{4}{|l|}{ Filhos } \\
\hline Sim & $231(26,4 \%)$ & $157(27,2 \%)$ & $74(24,8 \%)$ \\
\hline Não & $645(73,6 \%)$ & $421(72,8 \%)$ & $224(75,2 \%)$ \\
\hline Número de filhos M (DP) & $1,80(0,88)$ & $1,71(0,78)$ & $2,00(1,04)$ \\
\hline \multicolumn{4}{|l|}{ Religião/crença espiritual } \\
\hline Sim & $702(80,1 \%)$ & $497(86,0 \%)$ & $205(68,8 \%)$ \\
\hline Não & $174(19,9 \%)$ & $81(14,0 \%)$ & $93(31,2 \%)$ \\
\hline \multicolumn{4}{|l|}{ Qual religião/crença espiritual } \\
\hline $\begin{array}{l}\text { Sem religião, mas acredita em Deus (ou } \\
\text { alguma força superior) }\end{array}$ & $242(27,6 \%) \%$ & $167(28,9 \%)$ & $75(25,2 \%)$ \\
\hline Católica & $238(27,2 \%)$ & $171(29,6 \%)$ & $67(22,5 \%)$ \\
\hline Espírita & $111(12,7 \%)$ & $89(15,4 \%)$ & $23(7,7 \%)$ \\
\hline Evangélica & $67(7,6 \%)$ & $44(7,6 \%)$ & $22(7,4 \%)$ \\
\hline Umbanda & $6(0,7 \%)$ & $6(1,0 \%)$ & $0(0,0 \%)$ \\
\hline Judaica & $4(0,5 \%)$ & $2(0,3 \%)$ & $2(0,7 \%)$ \\
\hline Candomblé & $1(0,1 \%)$ & $1\left(0,2^{\%} \%\right)$ & $0(0,0 \%)$ \\
\hline Outra & $21(2,4 \%)$ & $12(2,1 \%)$ & $9(3,0 \%)$ \\
\hline \multicolumn{4}{|l|}{ Escolaridade } \\
\hline Ensino fundamental incompleto & $0(0,0 \%)$ & $0(0,0 \%)$ & $0(0,0 \%)$ \\
\hline Ensino fundamental completo & $2(0,2 \%)$ & $1(0,2 \%)$ & $1(0,3 \%)$ \\
\hline Ensino médio incompleto & $1(0,1 \%)$ & $0(0,0 \%)$ & $1(0,3 \%)$ \\
\hline Ensino médio completo & $13(1,5 \%)$ & $12(2,1 \%)$ & $1(0,3 \%)$ \\
\hline Ensino superior incompleto & $252(28,8 \%)$ & $151(26,1 \%)$ & $101(33,9 \%)$ \\
\hline Ensino superior completo & $152(17,4 \%)$ & $109(18,9 \%)$ & $43(14,4 \%)$ \\
\hline Pós-graduação incompleta & $100(11,4 \%)$ & $64(11,1 \%)$ & $36(12,1 \%)$ \\
\hline Pós-graduação completa & $356(40,6 \%)$ & $241(41,7 \%)$ & $115(38,6 \%)$ \\
\hline \multicolumn{4}{|l|}{ Trabalho } \\
\hline Sim & $639(72,9 \%)$ & $422(73 \%)$ & $217(72,8 \%)$ \\
\hline Não & $211(24,1 \%)$ & $137(23,7 \%)$ & $74(24,8 \%)$ \\
\hline Aposentado & $26(3,0 \%)$ & $19(3,3 \%)$ & $7(2,3 \%)$ \\
\hline
\end{tabular}

\section{Instrumentos}

Questionário de Sentido de Vida (MLQ - Steger, Frazier, Oishi, \& Kaler, 2006; versão brasileira adaptada por Damásio \& Koller, 2013): Instrumento composto por 10 itens, sendo cinco itens para avaliar presença de sentido (MLQ-P: "Minha vida tem um propósito claro") e cinco itens para avaliar busca de sentido (MLQ-S: "Eu estou buscando um objetivo ou missão para minha vida"). No presente estudo, foi utilizada apenas a escala Presença de sentido, cujos escores podem variar entre 5 e 35 . Neste estudo, a escala apresentou os seguintes índices de ajuste: CFI: 0,998; TLI = 0,996; RMSEA 0,075 (0,048 - 0106). O índice de consistência interna (alfa de Cronbach) foi de 0,91.

Escala de Felicidade Subjetiva (SHS - Lyubomirsky \& Lepper, 1999; versão brasileira adaptada por Damásio, Zanon, \& Koller, 2014). Instrumento unidimensional composto por quatro itens em escala tipo 
Likert variando de 1 a 7, que avalia os níveis de felicidade subjetiva. Os escores dos sujeitos podem variar de quatro a 28. Neste estudo, a escala apresentou os seguintes índices de ajuste: $\mathrm{CFI}=0,994$; $\mathrm{TLI}=0,982$; RMSEA $(90 \%$ CI $)=0,061(0,022-0,105)$. O índice de consistência interna (alfa de Cronbach) foi de 0,88.

Escala de Satisfação com a Vida (SWLS, Diener, Emmons, Larsen, \& Griffin, 1985; versão brasileira adaptada por Gouveia, Milfont, Fonseca, \& Coelho, 2009). É uma escala unidimensional Likert composta por cinco itens, cujas respostas podem variar de 1 (discordo totalmente) a 5 (concordo totalmente). Os escores dos sujeitos podem variar entre 5 e 25 pontos, sendo que escores mais altos correspondem a uma maior satisfação com a vida. Neste estudo, a escala apresentou os seguintes índices de ajuste: $\mathrm{CFI}=1,00$; TLI $=1,00$; RMSEA $(90 \% \mathrm{CI})=0,060(0,034-0,088)$; SRMR $=$ 0,011. O índice de consistência interna (alfa de Cronbach) foi de 0,89 .

Escala de Esperança para Adultos (AHS, Snyder et al., 1991; versão brasileira adaptada por Pacico, Bastianello, Zanon, \& Hutz, 2013). Escala tipo Likert (variando de $1=$ totalmente falso a $5=$ totalmente verdadeiro), composta por 12 itens, sendo quatro para avaliar o construto Agenciamento (i.e., senso de determinação para cumprir os objetivos com êxito) e quatro para avaliar o construto Rotas (i.e., capacidade de geração de planos de sucesso para atingir as metas propostas). As pontuações para ambas as escalas variam de 4 a 20 . O instrumento conta, também, com quatro itens de controle os quais não são considerados para análise. Neste estudo, a estrutura bidimensional da escala apresentou os seguintes índices de ajuste: $\mathrm{CFI}=0,95$, TLI $=0,93$, RMSEA $(90 \% \mathrm{CI})=0,083(0,070-0,097)$. Os índices de consistência interna (alfa de Cronbach) foram iguais a 0,81 tanto para agenciamento quanto para rotas.

Teste de Orientação de Vida (LOT-R - Scheier, Carver, \& Bridges, 1994; versão brasileira adaptada por Bastianello, Pacico, \& Hutz, 2014): A LOT-R avalia os níveis de otimismo (i.e., "Em tempos de incerteza, eu costumo esperar o melhor") e pessimismo (i.e., "Eu raramente espero coisas boas para mim") por meio de 10 itens respondidos em uma escala Likert de cinco pontos $(1=$ discordo totalmente; $5=$ concordo totalmente $)$. Para ambas as escalas, os escores podem variar de 4 a 20. Neste estudo, a estrutura bidimensional os seguintes índices de ajuste: $\mathrm{CFI}=0,98$, TLI $=0,96, \mathrm{RMSEA}(90 \%$ $\mathrm{CI})=0,067(0,047-0,089)$. Os índices de consistência interna (alfa de Cronbach) foram de 0,63 e 0,76 para as escalas Otimismo e Pessimismo, respectivamente.
Escala de Autoestima de Rosenberg (RSS; Rosenberg, 1989; versão brasileira adaptada por Hutz \& Zanon, 2011). Instrumento de 10 itens que tem por objetivo avaliar aspectos gerais da autoestima por meio de uma escala de quatro pontos tipo Likert $(1=$ discordo totalmente; $4=$ concordo totalmente). Os escores podem variar e 10 a 40, sendo que maiores escores correspondem a maiores níveis de autoestima. Neste estudo, foram encontrados os seguintes índices de ajuste: CFI $=0,93$, TLI $=0,91$, RMSEA $(90 \% \mathrm{CI})=0,090(0,080$ $-0,100)$. O índice de consistência interna (alfa de Cronbach) foi de 0,93 .

Escala General de Autoeficácia (GSS, Schwarzer \& Jerusalém, 1995; versão brasileira adaptada por Teixeira \& Dias, 2005): A GSS é uma escala unidimensional composta de 10 itens que avalia aspectos gerais relacionados à autoeficácia (sendo $1=$ não completamente verdadeiro e $7=$ exatamente verdadeiro). Os escores podem variar de 10 a 70, sendo maiores escores correspondentes a um maior senso de autoeficácia. Neste estudo, os índices de ajuste foram: CFI $=0,96$, TLI $=0,94$, RMSEA $(90 \% \mathrm{CI})=0,076(0,066-0,086)$. O índice de consistência interna (alfa de Cronbach) foi de 0,92.

Escala de Vitalidade Subjetiva (EVS, Bostic, Rubio, \& Hood, 2000; versão brasileira adaptada por Gouveia et al., 2012): A EVS é uma escala de sete itens, respondidos em escala tipo Likert de sete pontos, variando de $1=$ nada verdadeiro a $7=$ totalmente verdadeiro. $\mathrm{O}$ escore dos sujeitos pode variar de sete a 49 pontos, sendo que maiores escores representam maiores níveis de vitalidade subjetiva. Neste estudo, os índices de ajuste foram CFI $=0,98$, TLI $=0,97$, RMSEA $(90 \% \mathrm{CI})=0,082$ $(0,067-0,098)$. O índice de consistência interna (alfa de Cronbach) foi de 0,93.

Escalas de Depressão, Ansiedade e Estresse (DASS-21, Antony, Bieling, Cox, Enns, \& Swinson, 1998; versão brasileira adaptada por Machado, 2014): A DASS-21 é um instrumento de 21 itens desenvolvido para avaliar índices de depressão, ansiedade e estresse, sendo sete itens para cada construto. A escala apresenta um formato tipo Likert, com escores variando de 0 (nunca) a 3 (quase sempre). Para cada escala, os escores podem variar de 0 a 21, sendo que quanto maior o escore, maiores os níveis do sintoma avaliado. No presente estudo, a estrutura de três fatores da escala apresentou os seguintes índices de ajuste: $\mathrm{CFI}=0,96$, TLI $=0,96$, RMSEA $(90 \%$ CI $)=0,063(0,058-0,067)$. $\mathrm{O}$ índice de consistência interna (alfa de Cronbach) foi de 0,88, 0,82 e 0,87 para depressão, ansiedade e estresse, respectivamente. 


\section{Procedimentos de Coleta de Dados}

O presente estudo foi realizado após parecer favorável do Comitê de Ética em Pesquisa da Universidade Federal do Rio Grande do Sul (UFRGS) (protocolo $n^{\circ}$. 22240). A coleta de dados foi realizada por meio de uma página web desenvolvida especificamente para os objetivos da pesquisa. Para essa finalidade, foi utilizada a ferramenta SurveyMonkey, que consiste em um software, em formato de website, que tem por objetivo possibilitar a coleta de dados por meio de questionários on-line. Os contatos com os participantes foi realizados por e-mails $e$ por meio da técnica "bola de neve" (Patton, 1990). Todos participantes foram informados dos objetivos e procedimentos da pesquisa e a coleta de dados ocorreu mediante a concordância dos participantes que assinaram o Termo de Consentimento Livre e Esclarecido adicionado na primeira página da pesquisa.

\section{Procedimentos de Análise dos Dados}

\section{Invariancia da EP para Homens e Mulheres}

Inicialmente, foi realizada uma análise fatorial confirmatória multigrupo (AFCMG) para avaliar a invariância da EP para sexo $(n=578$ para mulheres e $n=298$ para homens). Três modelos de invariância foram testados. O modelo 1 (invariância configural) avaliou em que medida a estrutura fatorial da escala poderia ser considerada equivalente para os grupos. $\mathrm{O}$ modelo 2 (invariância métrica) avaliou o pressuposto da invariância nas cargas fatoriais do itens. Se esse modelo é aceito, isto significa que os itens apresentam relações semelhantes (i.e., a mesma importância) para o construto subjacente para todos os grupos (Brown, 2006; Damásio, 2013). O terceiro modelo (invariância escalar) avalia em que medida o intercepto dos itens são equivalentes para os grupos e garante que os escores obtidos estão totalmente relacionados com o nível de traço latente dos sujeitos, independente do seu grupo (Damásio, 2013; Milfont \& Fischer, 2010). A obtenção de invariância configural, métrica e escalar são requisitos indispensáveis para que comparações entre os grupos sejam adequadamente conduzidas. Isso porque, caso esses pressupostos não sejam satisfeitos, quaisquer diferenças encontradas entre os grupos podem estar relacionadas não a diferenças reais nos níveis do traço latente dos sujeitos, mas à não equivalência nos parâmetros do instrumento (Chen, 2008; Damásio, 2013).

A AFCMG foi avaliada utilizando o método de estimação Weighted Least Squares Mean and Variance-Adjusted (WLSMV; Muthén \& Muthén, 2010). O
WLSMV é implementado em uma matriz policórica e considera a natureza ordinal dos dados. Além disso, não requer distribuição de normalidade para os dados. A adequação da invariância configural foi avaliada por meio dos seguintes índices de ajuste: Comparative Fit Index (CFI), Tucker-Lewis Index (TLI) e Root Mean-Square Error of Approximation (RMSEA). De acordo com as diretrizes utilizadas (Brown, 2006), os valores de CFI e TLI devem estar acima de 0,90 (preferivelmente, acima de 0,95). Valores de SRMR e RMSEA devem estar abaixo de 0,08 , sendo que, para o RMSEA, o limite superior do intervalo de confiança $(90 \%)$ deve encontrar-se abaixo de 0,10 .

Para testar a invariância métrica e escalar, foi utilizado o teste de diferença do CFI ( $\triangle \mathrm{CFI}$; Cheung \& Rensvold, 2002). Diferenças no valor de CFI abaixo de 0,01 entre o modelo configural e os modelos subsequentes indicam que as cargas fatoriais e os interceptos dos itens podem ser considerados equivalentes para os grupos. Caso a diferença no CFI seja acima de 0,01, isso significa dizer que um ou mais parâmetros (cargas fatoriais e/ou interceptos) dos itens não são equivalentes entre os grupos, sugerindo algum tipo de viés de resposta a um ou mais itens (Damásio, 2013). Neste estudo, espera-se que haja invariância/equivalência do instrumento para todos os parâmetros testados.

\section{Evidências de Validade de Construto}

Com o objetivo de apresentar novas evidências de validade de construto para a EP, foram realizadas duas regressões lineares múltiplas (método Stepwise backward). A primeira regressão linear múltipla foi realizada inserindo a EP como variável dependente e medidas de Autoestima, Esperança (rotas e agenciamento), Satisfação com a vida, Otimismo e Pessimismo (variáveis constituintes do construto Positividade), além de Sentido de vida, Autoeficácia, Felicidade subjetiva, Vitalidade subjetiva, Estresse, Depressão e Ansiedade. É esperado que as variáveis constitutivas da Positividade (Autoestima, Satisfação com a vida, Otimismo e Esperança) sejam os mais fortes preditores dos escores da EP, explicando a maior parte da variância desse construto. Tal resultado deverá confirmar a hipótese de Caprara et al. (2009) e Caprara (2012, julho) de que a positividade é um compósito dessas variáveis.

O segundo modelo de regressão testou a influência das variáveis Autoeficácia, Sentido de vida, Vitalidade subjetiva, Felicidade subjetiva, Estresse, Depressão e Ansiedade, quando da ausência das variáveis constitutivas da EP. Tal análise foi realizada com o objetivo de 
investigar a influência dessas variáveis convergentes nos escores da EP sem a interferência da presença das variáveis constituintes do compósito Positividade.

As análises de regressão foram realizadas em detrimento das análises clássicas de correlação bivariada (Pearson ou Spearman) por possibilitar a avaliação de uma ordem hierárquica de importância dos construtos nos escores da EP. Além disso, uma vez que os construtos utilizados neste estudo podem ser fortemente correlatos, as técnicas de correlação bivariada poderiam superestimar as correlações entre as variáveis por não considerar as múltiplas variâncias compartilhadas entre si (Tabachnick \& Fidell, 2001). O método de regressão linear múltipla stepwise foi implementado haja vista que se busca avaliar um modelo teórico previamente estabelecido. Ademais, quando comparado com a técnica enter, o método stepwise diminui a incidência de problemas de multicolinearidade das variáveis (Field, 2005). A escolha da forma backward, por sua vez, se deu com o objetivo de evitar possíveis efeitos supressores (supressor effects), no qual uma variável só apresenta efeito significativo quando outra variável é inserida.

\section{Resultados}

\section{Análise Fatorial Confirmatória Multigrupo (AFMCG)}

Inicialmente, buscamos avaliar os índices de adequação de ajuste da Escala de Positividade separadamente para homens e para mulheres. Conforme pode ser visto na Tabela 2, o modelo inicial para ambos os sexos apresentou índices de ajuste adequado, exceto para o RMSEA, que acusou elevado níveis residuais em ambas as amostras. Uma avaliação pormenorizada do modelo, por meio dos índices de modificação, demonstrou, tanto para homens quanto para mulheres, uma significativa correlação residual entre os itens 1 ("Eu tenho muita confiança no futuro") e 6 ("Eu vejo o futuro com esperança e entusiasmo") ( $\mathrm{MI}=137,608$, $p<0,001$, para homens; $\mathrm{MI}=114,330, p<0,001$ para mulheres). A correlação entre os erros dos itens 1 e 6 foi de $r=0,61$ para a amostra masculina, e de $r=0,49$ para a amostra feminina. Considerando essa modificação no modelo, os índices de ajuste apresentam melhorias consideráveis (ver Tabela 2).

Após essa avaliação dos modelos de referência para homens e para mulheres, foi procedida a AFCMG para sexo. Num primeiro passo, testou-se a possibilidade de invariância configural, avaliando até que ponto o modelo final poderia ser considerado equivalente para homens e mulheres. Os índices de ajuste dessa configuração inicial foram bastante adequados, sugerindo sua plausibilidade (ver Tabela 2).

Posteriormente, procedeu-se a avaliação da invariância métrica e escalar, fixando todas as cargas fatoriais e interceptos dos itens para serem invariantes para homens e mulheres. O resultado desse modelo também foi bastante aceitável. $\mathrm{O}$ teste de diferença do CFI $(\Delta C F I<0,01)$ apresentou equivalência para ambos os parâmetros do instrumento, assegurando que a EP foi invariante para homens e para mulheres.

\section{Validade de Construto}

Com o objetivo de testar a validade de construto da EP, foram inseridas no primeiro modelo de regressão 13 variáveis (otimismo/pessimismo, esperança (agenciamento e rotas), satisfação com a vida,

Tabela 2

Índices de Adequação de Ajuste da Escala de Positividade para Sexo e para Modelos Multigrupo

\begin{tabular}{|c|c|c|c|c|c|}
\hline \multirow{2}{*}{\multicolumn{2}{|c|}{ Modelos individuais }} & \multicolumn{4}{|c|}{ Índices de adequação de ajuste } \\
\hline & & RMSEA (90\% IC) & TLI & CFI & $\Delta \mathrm{CFI}$ \\
\hline \multirow{2}{*}{ Homens } & Original & $0,169(0,147-0,191)$ & 0,951 & 0,965 & - \\
\hline & Modificado & $0,081(0,056-0,101)$ & 0,989 & 0,992 & - \\
\hline \multirow{2}{*}{ Mulheres } & Original & $0,142(0,127-0,158)$ & 0,965 & 0,975 & - \\
\hline & Modificado & $0,108(0,092-0,125$ & 0,980 & 0,986 & - \\
\hline \multirow{2}{*}{\multicolumn{2}{|c|}{ Modelos multigrupo }} & \multicolumn{4}{|c|}{ Índices de Adequação de Ajuste } \\
\hline & & RMSEA $(90 \%$ IC) & TLI & CFI & $\Delta \mathrm{CFI}$ \\
\hline \multirow{2}{*}{\multicolumn{2}{|c|}{$\begin{array}{c}\text { Invariância Configural } \\
\text { Invariância Métrica e Escalar }\end{array}$}} & $0,074(0,063-0,086)$ & 0,990 & 0,990 & - \\
\hline & & $0,063(0,053-0,074)$ & 0,992 & 0,993 & 0,00 \\
\hline
\end{tabular}

Nota. CFI - Comparative Fit Index; TLI - Tucker-Lewis Index, RMSEA - Root Mean Square Error of Approximation; IC - Intervalo de Confiança. 
autoestima, sentido de vida, felicidade subjetiva, vitalidade subjetiva, autoeficácia, depressão, estresse e ansiedade). Destas, otimismo, esperança, autoestima e satisfação com a vida são variáveis constitutivas do construto Positividade. O modelo proposto explicou 81,2\% dos escores da EP. Desse total, 79,4\% refere-se às variáveis centrais da Positividade: autoestima (61\%), satisfação com a vida $(11,2 \%)$, otimismo $(4,3 \%)$ e esperança (agenciamento, 2,9\%). As variáveis restantes do modelo (sentido de vida, vitalidade subjetiva, autoeficácia, estresse e felicidade subjetiva) explicaram apenas 1,8\% da variância restante dos escores da EP. Quatro variáveis inseridas não apresentaram incremento significativo no modelo: esperança (rotas; $p=0,49$ ), pessimismo $(p=0,07)$, depressão $(p=0,37)$ e ansiedade $(p=0,65)$. É importante salientar, porém, que o fato das variáveis Sentido de vida, Vitalidade, Autoeficácia, Estresse e Felicidade apresentarem baixa explicação na variância dos escores da EP, não significa que elas não sejam variáveis importantes para a compreensão do construto positividade. $\mathrm{Na}$ verdade, esse resultado inicial era esperado, visto que, propositalmente, foram inseridos no modelo I os componentes constitutivos do construto Positividade (Autoestima, Satisfação com a vida, Esperança e Otimismo), que explicaram quase que a totalidade da variância nos escores da EP.

Para dirimir dúvidas sobre a importância das outras variáveis na explicação dos escores de positividade e para atestar a validade convergente do construto, foi realizada uma nova regressão linear múltipla (método Stepwise backward). Nesse segundo modelo, foram retiradas as variáveis constituintes da positividade (Autoestima, Satisfação com a vida, Esperança e Otimismo), e inseridas as variáveis Sentido de vida, Vitalidade, Autoeficácia, Estresse e Felicidade (variáveis com pouca variância explicada no modelo 1), além de Esperança (rotas), Pessimismo, Depressão e Ansiedade (variáveis não significativas no modelo 1). Os resultados encontrados na Tabela 4 demonstram que as variáveis inseridas explicaram 71,5\% dos escores da EP. A Vitalidade explicou $53,7 \%$ dos escores da EP, enquanto Autoeficácia explicou 8,7\%; felicidade, 5,6\%; Sentido de vida, 2,9\% e Depressão 1,1\% (em sentido inverso). Ansiedade e Estresse não apresentaram associações significativas ( $\not=0,87$ e $p=0,60$, respectivamente).

\section{Discussão}

Inicialmente, buscou-se avaliar em que medida a estrutura fatorial da EP poderia ser considerada invariante para homens e mulheres. Para tanto, foram testados diversos modelos de invariância (configural, métrica e escalar) com o objetivo de atestar que a estrutura fatorial da EP, bem como as cargas fatoriais e interceptos dos itens poderiam ser considerados equivalentes para os dois grupos. Conforme esperado, os resultados corroboraram as hipóteses prévias de que a EP pode ser utilizada indistintamente para homens e mulheres, sem vieses de resposta. Entretanto, um aspecto importante necessita ser mencionado. Neste estudo, índices de modificação sugeriram que os erros dos itens 1 ("Eu tenho muita confiança no futuro") e 6 ("Eu vejo o futuro com esperança e entusiasmo") apresentaram significativa covariância entre si, indicando sobreposição de conteúdo entre esses indicadores (Brown, 2006) que, por sua vez, remetem a uma expectativa positiva

Tabela 3

Validade de Construto da Escala de Positividade com Variáveis Constitutivas e Convergentes

\begin{tabular}{lccccc}
\hline Modelo & $\mathrm{R}$ & $\mathrm{R}^{2}$ Ajustado & Modificação R & Beta & Sig. \\
\hline Autoestima & 0,781 & 0,610 & 0,610 &, 209 & 0,000 \\
Satisfação com a vida & 0,850 & 0,722 & 0,112 &, 199 & 0,000 \\
Otimismo & 0,875 & 0,765 & 0,043 &, 179 & 0,000 \\
Esperança (agenciamento) & 0,891 & 0,794 & 0,029 &, 194 & 0,000 \\
Sentido de vida & 0,896 & 0,803 & 0,009 &, 102 & 0,000 \\
Vitalidade & 0,899 & 0,808 & 0,005 &, 083 & 0,000 \\
Autoeficácia & 0,900 & 0,810 & 0,002 &, 066 & 0,001 \\
Estresse & 0,901 & 0,811 & 0,001 &,- 036 & 0,020 \\
Felicidade & 0,901 & 0,812 & 0,001 &, 054 & 0,035 \\
\hline
\end{tabular}

Nota. Modificação $R^{2}$ refere-se à contribuição individual de cada variável no modelo. 
Tabela 4

Validade de Construto da Escala de Positividade com Variáveis Convergentes

\begin{tabular}{lccccc}
\hline Modelo & $\mathrm{R}$ & $\mathrm{R}^{2}$ Ajustado & Modificação R & Beta & Sig. \\
\hline Vitalidade & 0,733 & 0,537 & 0,537 & 0,215 & 0,000 \\
Autoeficácia & 0,790 & 0,624 & 0,087 & 0,253 & 0,000 \\
Felicidade & 0,825 & 0,680 & 0,056 & 0,241 & 0,000 \\
Sentido de vida & 0,842 & 0,709 & 0,029 & 0,212, & 0,000 \\
Depressão & 0,846 & 0,715 & 0,006 & $-0,110$ & 0,000 \\
\hline
\end{tabular}

Nota. Modificação $R^{2}$ refere-se à contribuição individual de cada variável no modelo.

frente ao futuro. Esse resultado corrobora achados prévios (Borsa, Damásio, Souza, Koller, \& Caprara, 2015), onde os autores também encontraram covariância entre os erros desses itens. Considerando que dos oito itens da EP, três referem-se a expectativas positivas frente ao futuro (mesclando conteúdos de otimismo e esperança), e considerando que esses indicadores têm apresentado evidências de sobreposição de conteúdo, é possível que versões mais reduzidas da EP possam tornar a escala ainda mais parcimoniosa, especificamente, no que se refere aos itens que compreendem os construtos de esperança e otimismo.

Em relação às evidências de validade de construto, os modelos de regressão corroboraram as expectativas estabelecidas. Especificamente, o primeiro modelo evidenciou que a Positividade é, de fato, um construto composto pelas variáveis Autoestima, Satisfação com a vida, Otimismo e Esperança (Caprara \& Steca, 2005, 2006; Caprara et al., 2009; Caprara et al., 2010; Kozma, Stine, \& Stone, 2000), haja vista que essas variáveis explicaram quase que $80 \%$ dos escores da EP, mesmo quando inseridos um grande número de outras variáveis correlatas (Sentido de vida, Vitalidade, Felicidade subjetiva, Autoeficácia, Estresse, Depressão e Ansiedade), que explicaram apenas 1,8\% dos escores da EP. O fato de a Positividade ser comprovadamente um compósito dessas variáveis traz diversos benefícios às pesquisas científicas que tenham por objetivo investigar essas características pessoais. A EP configura-se como um instrumento curto, de rápida e fácil aplicação, psicometricamente confiável que, ao ser aplicado, traz informações sobre um conjunto importante de variáveis psicológicas (Caprara et al., 2009; Caprara, 2012, julho). Ao invés de utilizar diversos questionários, pesquisadores podem resumir suas baterias de pesquisa, empregando a EP em seus estudos, haja vista que, teórica e empiricamente, a EP engloba todos os indicadores anteriormente mencionados, formando uma dimensão única.
O modelo 2, por sua vez, confirmou a relação da positividade com outras variáveis positivas (validade convergente), corroborando diversos estudos conduzidos no âmbito da Psicologia Positiva (Alessandri, Caprara, \& Tisak, 2011; Alessandri, Caprara, \& Tisak, 2012; Caprara et al., 2010). Pode-se inferir, assim, que a disposição geral do indivíduo para avaliar positivamente os diversos aspectos da vida (positividade) estaria associada a uma concepção positiva sobre si mesmo, sobre o futuro e sobre as relações com as pessoas. Nesse segundo modelo, o percentual de variância explicada foi de $71,2 \%$, sendo que essas mesmas variáveis, no modelo 1, explicaram apenas 1,8\% do construto. Essa discrepância mostra o quanto as variáveis constitutivas da positividade (Satisfação com a vida, Autoestima, Esperança e Otimismo) são, de fato, capazes de predizer o construto, enquanto outras variáveis correlatas perdem o seu poder de explicação quando inseridas todas em um único modelo.

A variável mais fortemente preditora de positividade, nesse segundo modelo, foi a Vitalidade subjetiva, com variância compartilhada de 53,7\%. A Vitalidade subjetiva refere-se a um estado de vigor psicológico (também compreendido como um sentimento de vivacidade ou energia pessoal; Ryan \& Frederick, 1997) que é amplamente correlacionado com diversos indicadores do bem-estar (Gouveia et al., 2012), estando associado, entre outros aspectos, com a autorregulação do comportamento e com desfechos positivos ao longo do ciclo vital (Ryan \& Frederick, 1997). A alta associação entre a Positividade e a Vitalidade subjetiva é um forte indicativo de que pessoas com maiores níveis de positividade apresentam, também, melhor desenvolvimento em diversos aspectos de suas vidas. Embora essas associações sejam teoricamente plausíveis, futuros estudos devem avaliar longitudinalmente o impacto da positividade nos níveis de bem-estar e em diversas outras áreas 
da vida dos sujeitos, bem como investigar a estabilidade desse construto.

Este estudo apresenta algumas limitações. Inicialmente, não se contou com uma amostra representativa da população brasileira, de modo que futuros estudos são necessários para corroborar ou refutar os resultados aqui apresentados. Devido às características de distribuição da amostra em relação à idade, não foi possível avaliar a invariância da EP para diferentes grupos etários. De qualquer modo, o fato de a escala ter apresentado bons índices de ajuste para amostras variando de 18 a 83 anos é um indicativo inicial de que a medida pode ser utilizada com jovens, adultos e idosos.

\section{Considerações Finais}

O presente estudo teve por objetivo investigar novas evidências de validade para a Escala de Positividade (EP) para o contexto brasileiro. A EP tem sido utilizada em diferentes contextos culturais, apresentando invariância estrutural em países como Itália, Canadá, Alemanha e Japão (Caprara et al., 2011; Caprara et al., 2012b). No Brasil, a EP apresentou evidências iniciais de validade (Borsa et al., 2015). Entretanto, segundo as recomendações do Standards for educational and psychological testing (AERA; APA; NCME, 1999), é importante buscar evidências contínuas de validade para um instrumento psicológico. Neste estudo, foi demonstrado que a EP é uma medida confiável para ser utilizada tanto em homens quanto em mulheres, além de ter sido comprovado que a estrutura subjacente da EP engloba os construtos de autoestima, satisfação com a vida, esperança e otimismo, sendo, portanto, uma medida curta, confiável e de fácil administração para avaliar todos esses construtos em uma única escala.

\section{Referências}

Alessandri, G., Caprara, G. V., \& Tisak, J. (2011). The contribution of positive orientation to well adjustment during emerging adulthood. Manuscrito submetido para publicação.

Alessandri, G., Caprara, G. V., \& Tisak, J. (2012). The unique contribution of positive orientation to optimal functioning: Further explorations. European Psychologist, 17(1), 44-54. doi:10.1027/1016-9040/ a000070

American Educational Research Association [AERA], the American Psychological Association [APA] and the National Council on Measurement in
Education [NCME] (1999). Standards for educational and psychological testing. Washington, DC: American Educational Research Association.

Antony, M. M., Bieling, P. J., Cox, B. J., Enns, M. W., \& Swinson, R. P. (1998). Psychometric properties of the 42-item and 21-item versions of the Depression Anxiety Stress scales (DASS) in clinical groups and a community sample. Psychological Assessment, 10(2), 176-181. Recuperado de http://psycnet.apa.org/index.cfm?fa=buy. optionToBuy\&id=1998-02891-012

Bastianello, M. R., C., Pacico, J. C, \& Hutz, C. S. (2014). Optimism, self-esteem, and personality: Adaptation and validation of the Revised Life Orientation Test LOT-R. Psico-USF, 19(3), 523-531. doi: 10.1590/1413-827120140190030

Borsa, J. C., Damásio, B. F., Souza, D. S., Koller, S. H., \& Caprara, G. V. (2015). Psychometric properties of the brazilian version of the positivity Scale (PScale). Psicologia: Reflexão \& Crítica, 28(1), 61-67. doi:10.1590/1678-7153.201528107

Bostic, T. J., Rubio, D. M., \& Hood, M. (2000). A validation of the subjective vitality scale using structural equation modeling. Social Indicators Research, 52(3), 313-324. doi:10.1023/A:1007136110218

Brown, T. A. (2006).Confirmatory factor analysis for applied research. New York: The Guilford Press.

Caprara, G. V. (2012, Julho). Keynote at European Conference of Personality Psychology, Trieste.

Caprara, G. V., Alessandri, G., \& Barbaranelli, C. (2010). Optimal functioning: The contribution of self-efficacy beliefs to positive orientation. Psychotherapy and Psychosomatics, 79, 328-330. doi: 10.1159/000319532

Caprara, G. V., Alessandri, G., Eisenberg, N., Kupfer, A., Steca, P., Caprara, M. G.,... Abela, J. (2012a). The positivity scale. Psychological Assessment, 24(3), 701-712. doi:10.1037/a0026681

Caprara, G. V., Alessandri, G., Trommsdorff, G., Heikamp, T., Yamaguchi, S., \& Suzuki, F. (2012b). Positive orientation across three cultures. Journal of Cross-Cultural Psychology, 43(1), 77-83. doi:10.1177/0022022111422257

Caprara, G. V., Fagnani, C., Alessandri, G., Steca, P., Gigantesco, A., Cavalli Sforza, L. L., \& Stazi, M. A. (2009). Human optimal functioning: The genetics of positive orientation towards self, life, 
and the future. Behavior Genetics, 39(3), 277-284. doi:10.1007/s10519-009-9267-y

Caprara, G. V. \& Steca, P. (2005). Affective and social self-regulatory efficacy beliefs as determinants of positive thinkingand happiness. European Psychologist, 10(4), 275-286. doi: 10.1027/1016-9040.10.4.275

Caprara, G. V. \& Steca, P. (2006). The contribution of self-regulatory efficacy beliefs in managing affect and family relationships to positive thinking and hedonic balance. Journal of Social and Clinical Psychology, 25(6), 601-625. doi: 10.1521/ jscp.2006.25.6.603

Caprara, G. V., Steca, P., Alessandri, G., Abela, J. R. Z., \& McWhinnie, C. M. (2010). Positive orientation: Explorations on what is common to life satisfaction, self-esteem, and optimism. Epidemiologia e Psichiatria Sociale, 19(1), 63-71. doi:10.1017/ S1121189X00001615

Carver, C. S., Scheier, M. F., \& Segerstrom, S. C. (2010). Optimism. Clinical Psychology Review, 30(7), 879-889. doi:10.1016/j.cpr.2010.01.006

Chen, F. F. (2008). What happens if we compare chopsticks with forks? The impact of making inappropriate comparisons in cross-cultural research. Journal of Personality and Social Psychology, 95(5), 1005-1018. doi:10.1037/a0013193.

Cheung, G. W., \& Rensvold, R. B. (2002). Evaluating goodness-of-fit indexes for testing measurement invariance. Structural Equation Modeling: A Multidisciplinary Journal, 9(2), 233-255. doi:10.1207/ S15328007SEM0902_5

Damásio, B. F. (2013). Contribuições da análise fatorial confirmatória multigrupo (AFCMG) na avaliação de invariância de instrumentos psicométricos. Psico-USF, 18(2), 211-220. doi:10.1590/ S1413-82712013000200005

Damásio, B. F., \& Koller, S. H. (2013). Validation and Psychometric Properties of the Brazilian Version of the Meaning in Life Questionnaire. Manuscrito submetido para publicação.

Damásio, B. F., Zanon, C., \& Koller, S. H. (2014). Validation and psychometric properties of the Brazilian version of the subjective happiness scale. Universitas Psychologica, 13(1), 1-13. doi:10.11144/ Javeriana.UPSY13-1.vppb
Diener, E. (2013). The remarkable changes in the science of subjective well-being. Perspectives on Psychological Science, 8(6), 663-666. doi:10.1177/1745691613507583

Diener, E., Emmons, R. A., Larsen, R. J., \& Griffin, S. (1985). The satisfaction with life scale. Journal of Personality Assessment, 49(1), 71-75. doi: 10.1207/ s15327752jpa4901_13

Diener, E., Scollon, C. K. N., Oishi, S., Dzokoto, V., \& Suh, E. M. (2000). Positivity and the construction of life satisfaction judgments: Global happiness is not the sum of its part. Journal of Happiness Studies, 1(2), 159-176. doi:10.1023/A:1010031813405

Field, A. (2005). Discovering Statistics Using SPSS (2nd ed.). London: Sage Publications.

Fredrickson, B. L. (2009). Positivity: Groundbreaking research reveals how to embrace the bidden strength of positive emotions, overcome negativity, and thrive. New York: Crown.

Gable, S., \& Haidt, J. (2005). What (and Why) is positive psychology? Review of General Psychology, 9(2), 103110. doi:10.1037/1089-2680.9.2.103

Gouveia, V. V., Milfont, T., Fonseca, P. N., \& Coelho, J. A., P. M. (2009). Life satisfaction in Brazil: Testing the psychometric properties of the Satisfaction With Life Scale (SWLS) in five Brazilian samples. Social Indicators Research, 90(2), 267-277. doi:10.1007/s11205-008-9257-0

Gouveia, V. V., Milfont, T. L., Gouveia, R. S. V., Medeiros, E. D., Vione, K. C., \& Soares, A. L. S. (2012). Escala de vitalidade subjetiva - EVS: Evidências de sua adequação psicométrica. Psicologia: Teoria e Pesquisa, 28(1), 5-13. doi: 10.1590/ S0102-37722012000100002

Hutz, C. S., \& Zanon, C. (2011). Revisão da adaptação, validação e normatização da escala de autoestima de Rosenberg. Avaliação Psicológica, 10(1), 41-49. Recuperado de http://pepsic.bvsalud.org/pdf/avp/ v10n1/v10n1a05.pdf

Jayawickreme, E., \& Pawelski, J. O. (2012). Positivity and the capabilities approach. Philosophical Psychology. doi:10.1080/09515089.2012.66068

Kozma, A., Stone, S., \& Stones, M. J. (2000). Stability in components and predictors of subjective well-being (SWB): Implications for SWB structure. Em E. Diener and D. R. Rahtz (Eds.), 
Advances in quality of life theory and research (Vol. 1, pp. 13-30). London, England: Kluwer Academic. doi:10.1007/978-94-011-4291-5_2

Lyubomirsky, S., \& Lepper, H. S. (1999). A measure of subjective happiness: Preliminary reliability and construct validation Social Indicators Research, 46(1), 137-155. doi:10.1023/A:1006824100041

Machado, W. L. (2014). A saúde mental positiva e sua relação com indicadores de psicopatologia, personalidade, qualidade de vida e características sociodemográficas (Tese de doutorado não-publicada). Curso de Pós-Graduação em Psicologia da Universidade Federal do Rio Grande do Sul. Porto Alegre.

Milfont, T. L., \& Fischer, R. (2010). Testing measurement invariance across groups: Applications in cross-cultural research. International Journal of Psychological Research, 3(1), 111-121. Recuperado de http:/ / www.redalyc.org/articulo.oa?id $=299023509008$

Muthén, L. K., \& Muthén, B. O. (2010). Mplus: Statistical analysis with latent variables. User's guide. Los Angeles: Muthén \& Muthén.

Pacico, J. C., Bastianello, M. R., Zanon, C., \& Hutz, C.S. (2013). Adaptation and validation of the dispositional hope scale for adolescents. Psicologia: Reflexão e Crítica, 26(3), 488-492. doi: 10.1590/ S0102-79722013000300008

Patton, M. Q. (1990). Qualitative evaluation and research methods ( $2^{\circ}$ ed.), Newbury Park: Sage.

Rosenberg, M. (1989). Society and the adolescent self-image. Revised Edition. Middletown, CT: Wesleyan University Press.

Ryan, R. M., \& Frederick, C. M. (1997). On energy, personality and health: Subjective vitality as a dynamic reflection of well-being. Journal of Personality, 65(3), 529-565. doi: 10.1111/j.1467-6494.1997.tb00326.x

Scheier, M. F., \& Carver, C. S. (1993). On the power of positive thinking: The benefits of being optimistic. Current Directions in Psychological Science, 2, 26-30. doi:10.1111/1467-8721.ep10770572

Scheier, M. F., Carver, C. S., \& Bridges, M. W. (1994). Distinguishing optimism from neuroticism (and trait anxiety, self-mastery, and self-esteem) - a reevaluation of the Life Orientation Test. Journal of Personality and Social Psychology, 67(6), 1063-1078. doi:10.1037/0022-3514.67.6.1063
Schwarzer, R., \& Jerusalem, M. (1995). Generalized self-Efficacy scale. Em J. Weinman, S. Wright, \& M. Johnston (Eds.), Measures in health psychology: $A$ user's porffolio. Causal and control beliefs (pp. 35-37). Windsor, UK: NFER-NELSON.

Seligman, M. E. P., \& Csikszentmihalyi, M. (2000). Positive psychology: An introduction. American Psychologist, 55, 5-14. doi: 10.1037/0003-066X.55.1.5

Snyder, C. R., Harris, C., Anderson, J. R., Holleran, S. A., Irving, L. M., Sigmon, S. X.,... , Harney, P. (1991). The will and the ways: Development and validation of an individual-differences measure of hope. Journal of Personality and Social Psychology, 60(4), 570-585. Recuperado de http://www.ncbi. nlm.nih.gov/pubmed/2037968

Snyder, C. R., Rand, K. L., \& Sigmon, D. R. (2002). Hope theory: A member of the positive psychology family. Em C. R. Snyder \& S. J. Lopez, (Eds.), Handbook of positive psychology (pp.257-276). New York: Oxford University Press.

Steger, M. F., Frazier, P., Oishi, S., \& Kaler, M. (2006). The meaning in life questionnaire: Assessing the presence of and search for meaning in life. Journal of Counseling Psychology, 53(1), 80-93. doi:10.1037/0022-0167.53.1.80

Tabachnick, B. G., \& Fidel, L. S. (2001). Using multivariate statistics. San Francisco: Allyn \& Bacon.

Teixeira, M. A. P., \& Dias, A. C. G. (2005). Propriedades psicométricas da versão traduzida para o português da Escala de Autoeficácia Geral Percebida de Ralph Schwarzer [Psychometric properties of the Brazilian-portuguese translated version of the Ralph Schwarzer's Perceived Self-efficacy Scale]. Em Instituto Brasileiro de Avaliação Psicológica (Ed.), Abstracts of the Second Brazilian Congress of Psychological Assessment. Gramado: IBAP.

Urbina, S. (2004). Essentials of Psychological Testing. New Jersey: John Wiley \& Sons, Inc.

Werner, E. E. (2013). What can we learn about resilience from large-scale longitudinal studies? Em S. Goldstein \& R. B. Brooks. (Eds.), Handbook of Resilience in Children (2 ${ }^{\circ}$ ed.) (pp. 87-102). New York: Springer.

Recebido em: 03/06/2014

Primeira reformulação em: 16/01/2015

Aprovado em: 05/02/2015 
Nota dos autores:

Apoio CNPq.

Sobre os autores:

Juliane Callegaro Borsa é psicóloga, mestre em Psicologia Clínica pela Pontifícia Universidade Católica do Rio Grande do Sul (PUCRS), doutora em Psicologia pela Universidade Federal do Rio Grande do Sul (UFRGS) e professora do Departamento de Psicologia da Pontifícia Universidade Católica do Rio de Janeiro (PUC-Rio).

E-mail: juliborsa@gmail.com

Bruno Figueiredo Damásio é psicólogo, mestre e doutor em Psicologia pela Universidade Federal do Rio Grande do Sul (UFRGS) e professor do Instituto de Psicologia, Departamento de Psicometria da Universidade Federal do Rio de Janeiro (UFRJ).

E-mail: bf.damasio@gmail.com

Silvia Helena Koller é psicóloga, mestre em Psicologia pela Universidade Federal do Rio Grande do Sul (UFRGS), doutora em Psicologia pela Arizona State University (ASU), Estados Unidos, doutora em Educação pela Pontifícia Universidade Católica do Rio Grande do Sul (PUCRS), professora do Instituto de Psicologia da Universidade Federal do Rio Grande do Sul (UFRGS) e pesquisadora 1A do CNPQ.

E-mail: silvia.koller@gmail.com

\section{Contato com os autores:}

Juliane Callegaro Borsa

Departamento de Psicologia da Pontifícia Universidade Católica do Rio de Janeiro (PUC-Rio).

Rua Marquês de São Vicente, n. 225. Edifício Cardeal Leme, sala 201, Gávea, Rio de Janeiro, RJ.

CEP: 22451-900 